\title{
Adaptive Fuzzy Control for Path Tracking of Underactuated Ships Based on Dynamic Equilibrium State Theory
}

\author{
Dehui Qiu, ${ }^{1,2}$, Qinglin Wang*, Jie Yang \\ ${ }^{1}$ School of Automation, Beijing Institute of Technology \\ Beijing, 100081, China \\ ${ }^{2}$ School of Information Engineering, Capital Normal University \\ Beijing, 100048, China \\ Jinhua She \\ School of Computer Science, Tokyo University of Technology \\ Tokyo, 192-0982, Japan \\ Received 20 August 2011 \\ Accepted 25 November 2011
}

\begin{abstract}
This paper presents an adaptive fuzzy backstepping control method that incorporates the dynamic equilibrium state (DES) theory to carry out path tracking for underactuated ships in presence of parameter variations and external disturbances induced by wind, wave and current. First, the optimal DES reference trajectories are designed for the sway displacement and the yaw angle. Then, by combining the DES theory with backstepping technology and using fuzzy logic systems to approximate unknown nonlinear functions in the system, a robust adaptive fuzzy controller is designed to track the prescribed trajectories. Theoretical analysis and simulation results show that the proposed method performs path tracking of underactuated ships with high precision, eliminates the influence of the uncertainties, and guarantees global stability and robustness of the system.
\end{abstract}

Keywords: underactuated ships, dynamic equilibrium state (DES), backstepping, fuzzy logic systems

\section{Introduction}

In recent years, the design of the trajectory tracking and the path following controllers for underactuated ships is one of the hot issues in many offshore applications. ${ }^{1}$ Many remarkable results have been achieved in the ship straight line tracking control. $^{2-7}$ However, an underactuated ship usually has big inertia, big time lag, nonlinearity and underactuated characteristics. And its tracking motion is strongly influenced by the model parameter perturbations, as well as the effects of wind, wave and current flow disturbances. So it is very necessary to design the tracking controller with strong robustness and high precision when there exist uncertainties from model and disturbance.

Wang et al. [8-10] addressed a kind of nonlinear tracking design methods based on the DES theory, which are established on the concept of the DES asymptotic stability. The DES is a type of equilibrium states targeted at the non-free system. It is not the origin or a certain fixed point but the function of the input. The concept of DES and its stability solve the conceptual conflict, which results from the application of the Lyapunov direct method to the tracking problem. Its

\footnotetext{
*Corresponding author: wqlbit@gmail.com
} 
most outstanding feature lies in the unification of the stability and tracking problems when the method makes the state of the system track its desired DES in the optimal way. The control scheme in Ref. 8-10 was only limited to the affine nonlinear systems, so this paper attempts to utilize this method to deal with the path tracking control of the underacutuated ships. The present paper firstly reports about the ship tracking control based on the DES theory.

Wang [11] presented a foundation of the adaptive fuzzy control where it combines the merits of both conventional fuzzy and adaptive control. Now, adaptive fuzzy control has been an effective control method for the uncertain nonlinear systems. ${ }^{12-17}$

In view of this, this paper combines the DES tracking approach with adaptive fuzzy backstepping control method to obtain the desired path tracking of underactuated ships in presence of parameters variations and external disturbances caused by wind, wave and current. The proposed adaptive fuzzy controller guarantees the stability of the closed-loop system and achieves the good tracking performance as well.

The rest of the chapter is arranged as follows: Section 2 introduces the concept of DES theory and tracking approach based on it. In section 3, the description of an underactuated ship system with parameter variations and external disturbances is given and the ideal DES tracking trajectories are designed. An adaptive fuzzy controller for the path tracking of underactuated ships is designed in section 4. The simulation results are provided in Section 5. The final conclusion is shown in section 6 .

\section{The DES theory}

The stability is the main performance index of control systems. But traditional stability theory targeted at the free system and isolated the equilibrium state from the input. The proposal of the DES concept opens a new way to study the stability of non-free systems with the input. The definition of a DES is as follows.

Definition 1: Consider a control system $\dot{x}=f(x, u(t), t)$, the steady-forced part $x_{e}(u(t))$ of the solution is called the system DES under the action of $u(t)$, and is also called the system dynamic equilibrium $x_{e}(t)$ for short. ${ }^{8}$

The DES refers to the non-free system's stability state. The introduction of the DES gives a new description to the dynamical relationships of the system. Classical control theory did not reveal the internal variables, but study the relationship between the input and the output. Modern control theory studied the relationships between input, state and output. The state space method played a key role in revealing and understanding many important characters of control systems. State controllability and observability are particularly important, which become two basic concepts of control theory. But the conceptions of state controllability and observability in modern control theory did not combine with system stability. The DES theory studies the relationships between the four factors: the input, the DES, the state and the output. The relationships can be shown as Fig. 1.

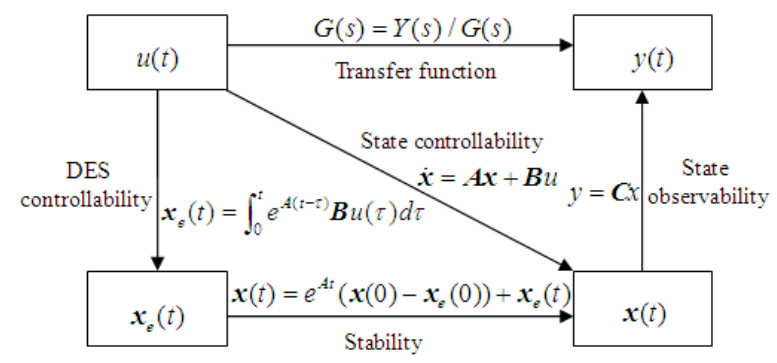

Fig. 1. The relationship between input, DES, state and output

From Fig. 1, it is seen that the relationship between the DES and the state, i.e. stability, can be revealed. The theory of the DES claims that what is controlled directly by the input of the control system is the DES rather than the state or the output. When a system is asymptotically stable to its DES in the large, the state or the output will asymptotically converge to the system dynamic equilibrium under the constraints the system structure. That is the basic idea of equilibrium state control. ${ }^{8}$

The tracking control method of the nonlinear systems based on the DES theory includes three procedures. Firstly, based on the quality index of the controlled systems, the linear time-invariant system with ideal dynamic properties is designed as reference model. Second, the state of the model is dealt with as the DES of the controlled system. Third, the control law is designed to make the state track to its DES, finally achieving globally asymptotic stability. Therefore the controlled systems approximately present the dynamic performance of the reference model, and the feedback linearization of nonlinear system is achieved. 


\section{Problem statement}

Fig. 2. is the straight-line trajectory keeping control model for the underactuated ships.

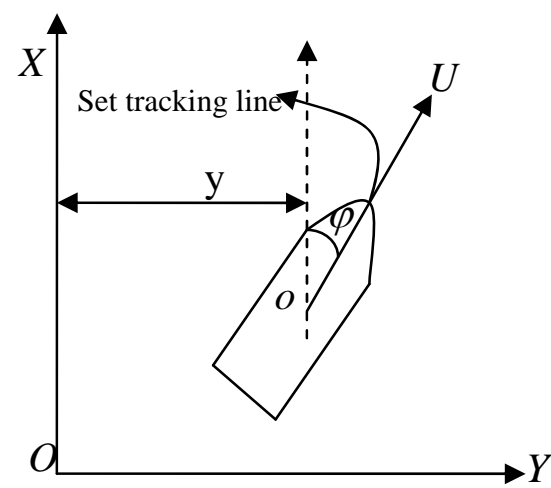

Fig. 2. Ship motion coordinate system

In Ref. 5, ship straight line trajectory keeping control model equation is described as follows:

$$
\left\{\begin{array}{l}
\dot{y}=U \sin \varphi+\omega_{1} \\
\dot{\varphi}=r \\
\dot{r}=-\frac{1}{T} r-\frac{\alpha}{T} r^{3}+\frac{K}{T} u+\omega_{2}
\end{array}\right.
$$

where $y, \varphi, r$ and $U$ denote the sway displacement, yaw angle, yaw rate and cruise speed respectively. $T$ and $K$ represent ship model operation index parameters. $\alpha$ is a constant parameter denoting the nonlinear item in the model. $u$ denotes the control rudder angle. $\omega_{1}$ and $\omega_{2}$ are external bounded disturbances.

To simplify the design process, the following coordinate transformation is defined according to Ref. 5. Such that

$$
s=k y+\varphi
$$

Where $k$ is a positive constant. The convergence of $S$ and $y$ to zero means that of $\varphi$.

Considering Eq. (2), Eq. (1) can be simplified as

$$
\left\{\begin{array}{l}
\dot{s}=k U \sin \varphi+r+\omega_{1} \\
\dot{r}=-\frac{1}{T} r-\frac{\alpha}{T} r^{3}+\frac{K}{T} u+\omega_{2}
\end{array}\right.
$$

Let $\quad x_{1}=y(t) \quad, \quad x_{2}=\varphi(t) \quad, \quad x_{3}=r(t) \quad$, $f_{1}=k U \sin \varphi, \quad f_{2}=-\frac{1}{T} r-\frac{\alpha}{T} r^{3}, \quad b_{1}=1, \quad b_{2}=\frac{K}{T}$, then the ship model Eq. (3) can be rewritten as follows

$$
\left\{\begin{array}{l}
\dot{s}=f_{1}+b_{1} x_{3}+\omega_{1} \\
\dot{x}_{3}=f_{2}+b_{2} u+\omega_{2}
\end{array}\right.
$$

In practice, because of the external disturbances, such as wind, wave and ocean current, the cruise speed is always time varying and $T, K$ and $\alpha$ are uncertain parameters. Then, $f_{1}, f_{2}$ are unknown nonlinear smooth functions; $b_{1}, b_{2}$ are unknown constants.

In order to design robust adaptive fuzzy controller, the following assumptions are given.

Assumption 1. There exist positive constants $b_{i m}$ and $b_{i M}$, such that $0<b_{i m} \leq\left|b_{i}\right| \leq b_{i M}, \quad i=1,2$.

Assumption 2. The external disturbances are bounded, such that $\omega_{i}^{2} \leq c_{i}$.

According to the stable state control of the DES theory, this paper uses the linear ship line tracking model to find the desired DES reference trajectories based on linear quadratic optimum control method.

Let $\sin \varphi=\varphi$, and the nonlinear item model parameter $\alpha$ is neglected. The approximate linearization states equations of the ship line tracking model Eq. (1) are as follows:

$$
\dot{\boldsymbol{x}}=\boldsymbol{A} \boldsymbol{x}+\boldsymbol{B} u=\left[\begin{array}{ccc}
0 & U & 0 \\
0 & 0 & 1 \\
0 & 0 & -1 / T
\end{array}\right]\left[\begin{array}{c}
x_{1} \\
x_{2} \\
x_{3}
\end{array}\right]+\left[\begin{array}{c}
0 \\
0 \\
K / T
\end{array}\right] u
$$

$\boldsymbol{Q}$ and $\boldsymbol{R}$ are chosen according to desired performance index. Then the linear quadratic optimal feedback control law is given by

$$
u=-\boldsymbol{K} \boldsymbol{x}
$$

Substituting Eq. (6) to Eq. (5) and adding input $r$, then the desired DES reference trajectories of the ship line tracking model can be expressed by the linear reference model as follows:

$$
\dot{\boldsymbol{x}}_{d}=\boldsymbol{A}_{d} \boldsymbol{x}_{d}+\boldsymbol{B}_{d} r=(\boldsymbol{A}-\boldsymbol{B K}) \boldsymbol{x}_{d}+\boldsymbol{B} k_{1} r
$$

where $k_{1}$ is the first component of $\boldsymbol{K} . \boldsymbol{x}_{d}$ is taken as the DES of ship line tracking control.

We can define $s_{d}=k x_{d 1}+x_{d 2} . s_{d}$ is taken as the DES of $s$. Then, the control objective is to make $s$ and 
$x_{3}$ track their DES $s_{d}$ and $x_{3 d}$. Finally, the tracking errors asymptotically converge to zero.

\section{Adaptive fuzzy backstepping control based on the DES}

\subsection{Backstepping control based on the DES}

The backstepping procedure is an efficient design approach for nonlinear systems with strict feedback structure. It consists of a step-by-step construction of a transformed system with $z_{i}=x_{i}-\alpha_{i}$, where $\alpha_{i}$ is the so-called virtual control at the step $i$. The virtual control $\alpha_{i}$, in fact , is the new DES of $x_{i}$, so the backstepping control method based on the DES in a sense can be explained by designing control law to let state to track and converge to its DES step by step, ultimately realizing global stability of the close loop system. The concept of virtual control is replaced with the DES, making the procedure more clear and easy to understand.

The procedures of the backstepping design based on the DES are given as follows:

Step 1: For the first subsystem $\dot{s}=f_{1}+b_{1} x_{3}+\omega_{1}$, the control law is designed to achieve state $s$ asymptotically converging to its DES. Let the tracking error $z_{1}=s-s_{d}$. Its derivative is

$$
\begin{aligned}
\dot{z}_{1} & =\dot{s}-\dot{s}_{d}=f_{1}+b_{1} x_{3}+\omega_{1}-\dot{s}_{d} \\
& =b_{1} z_{2}+b_{1} x_{d 3}+f_{1}+\omega_{1}-\dot{s}_{d}
\end{aligned}
$$

where the second tracking error $z_{2}=x_{3}-x_{d 3} \cdot x_{d 3}$ is taken as the new DES of $x_{3}$.

Define the first Lyapunov function candidate as

$$
\begin{gathered}
V_{1}=\frac{1}{2 b_{1}} z_{1}^{2} \\
\dot{V}_{1}=\frac{1}{b_{1}} z_{1}\left(b_{1} z_{2}+b_{1} x_{d 3}+f_{1}+\omega_{1}-\dot{s}_{d}\right) \\
=z_{1}\left(z_{2}+x_{d 3}\right)+\frac{1}{b_{1}} z_{1}\left(f_{1}-\dot{s}_{d}\right)+\frac{1}{b_{1}} z_{1} \omega_{1} \\
=z_{1}\left(z_{2}+x_{d 3}+\hat{f}_{1}\right)+\frac{1}{b_{1}} z_{1} \omega_{1}
\end{gathered}
$$

where $\hat{f}_{1}=\frac{1}{b_{1}} z_{1}\left(f_{1}-\dot{s}_{d}\right)$.
Let $x_{d 3}=-\lambda_{1} z_{1}-\tau_{1}\left(\lambda_{1}>0\right) . \quad \hat{\tau}_{1}$ is a fuzzy logic system to approximate the $\hat{f}_{1}$, such that

$$
\dot{V}_{1}=-\lambda_{1} z_{1}^{2}+z_{1} z_{2}+z_{1}\left(\hat{f}_{1}-\hat{\tau}_{1}\right)+\frac{1}{b_{1}} z_{1} \omega_{1}
$$

Step 2: The derivation of $z_{2}$ is

$$
\dot{z}_{2}=\dot{x}_{3}-\dot{x}_{d 3}=f_{2}+b_{2} u+\omega_{2}-\dot{x}_{d 3}
$$

Select the augmented Lyapunov function as

$$
V_{2}=V_{1}+\frac{1}{2 b_{2}} z_{2}^{2}
$$

Let $\hat{f}_{2}=\frac{1}{b_{2}} z_{1}\left(f_{2}-\dot{x}_{3 d}\right)$.The control law is formulated as

$$
u=-\lambda_{2} z_{2}-z_{1}-\hat{\tau}_{2}, \quad \lambda_{2}>0
$$

$\hat{\tau}_{2}$ is a fuzzy logic system to approximate the $\hat{f}_{2}$, such that :

$$
\begin{aligned}
\dot{V}_{2} & =\dot{V}_{1}+\frac{1}{b_{2}} z_{2} \dot{z}_{2} \\
& =\dot{V}_{1}+z_{2}\left(u+\frac{1}{b_{2}}\left(\hat{f}_{2}-\dot{x}_{3 d}\right)\right)+\frac{1}{b_{2}} z_{2} \omega_{2}
\end{aligned}
$$

Substituting Eq. (14) into above Eq. (15), we get

$$
\begin{aligned}
\dot{V}_{2}= & -\lambda_{1} z_{1}^{2}-\lambda_{2} z_{2}^{2}+z_{1}\left(\hat{f}_{1}-\tau_{1}\right) \\
& +z_{2}\left(u+z_{1}+\frac{1}{b_{2}}\left(\hat{f}_{2}-\dot{x}_{d 3}\right)\right)+\frac{1}{b_{1}} z_{1} \omega_{1}+\frac{1}{b_{2}} z_{2} \omega_{2}
\end{aligned}
$$

\subsection{Apative fuzzy control}

Because a fuzzy logic system has the capability of universal approximation for any unknown nonlinear functions to the desired accuracy in a compact set, two fuzzy estimators $\hat{\tau}_{1}$ and $\hat{\tau}_{2}$ are designed to approximate $\hat{f}_{1}$ and $\hat{f}_{2}$. A fuzzy logic system consists of four parts: the knowledge base, the fuzzifier, the fuzzy inference engine working on fuzzy rules, and the defuzzifier. The knowledge base for fuzzy logic system comprises a collection of fuzzy IF-THEN rules of the following form:

$R^{(l)}$ : If $x_{1}$ is $F_{1}^{l}$ and $x_{2}$ is $F_{2}^{l}, \cdots, \quad x_{N}$ is $F_{N}^{l}$, then $y \in G^{l} \quad l=1,2, \cdots, M$.

where $\boldsymbol{x}=\left[x_{1}, x_{2}, \cdots x_{N}\right]^{T}$ and $y$ are fuzzy logic system 
input and output, respectively, Fuzzy sets $F_{1}^{l}$ and $G^{l}$ associated with the fuzzy function $\mu_{F_{i}^{l}}\left(x_{i}\right)$ and $\mu_{G^{l}}(y)$. $M$ represents the rule number.

In this study, the fuzzy system is implemented with singleton fuzzification and product inference, and the defuzzifier is executed by the method of center of gravity. The input and output relation of the fuzzy system is obtained as ${ }^{11-14}$

$$
y(\boldsymbol{x}, \boldsymbol{\theta})=\frac{\sum_{l=1}^{M} \theta_{l}\left(\prod_{i=1}^{N} \mu_{F_{i}^{\prime}}\left(x_{i}\right)\right)}{\sum_{l=1}^{M}\left(\prod_{i=1}^{N} \mu_{F_{i}^{\prime}}\left(x_{i}\right)\right)}
$$

where $\theta_{l}=\max _{y \in R} G^{l}(y) \cdot \mu_{F_{i}^{l}}\left(x_{i}\right)$ is called Gaussian membership function of fuzzy set $F_{i}^{l} ; \boldsymbol{\theta}=\left[\theta^{1}, \cdots, \theta^{M}\right]^{T}$ is the optimal parameter vector; $\boldsymbol{\xi}(\boldsymbol{x})=\left[\boldsymbol{\xi}_{1}(\boldsymbol{x}), \cdots, \boldsymbol{\xi}_{M}(\boldsymbol{x})\right]^{T}$ is a fuzzy basis vector with the input vector $\boldsymbol{x}$. Then Eq. (17) can be rewritten as follows:

$$
y(\boldsymbol{x}, \boldsymbol{\theta})=\boldsymbol{\theta}^{T} \boldsymbol{\xi}(\boldsymbol{x})
$$

The inputs of fuzzy logic systems are designed as: $X_{1}=s$ and $X_{2}=x_{3}$.

Let $\bar{X}_{i}=\left[X_{1}, X_{2}, \cdots, X_{i}\right]^{T},(i=1,2)$, then

$$
\hat{\tau}_{1}=\hat{\theta}_{1}^{T} \xi\left(\bar{X}_{1}\right), \quad \hat{\tau}_{2}=\hat{\theta}_{2}^{T} \xi\left(\bar{X}_{2}\right)
$$

Based on the universal approximation theorem, ${ }^{11-12}$ the optimal fuzzy approximation $\hat{\tau}_{1}\left(\bar{X}_{1}, \theta_{1}^{*}\right)$ and $\hat{\tau}_{2}\left(\bar{X}_{2}, \boldsymbol{\theta}_{2}^{*}\right)$ are further designed to approximate the $\hat{\tau}_{1}$ and $\hat{\tau}_{2}$, such that

$$
\hat{f}_{i}=\hat{\tau}_{i}\left(\bar{X}_{i}, \boldsymbol{\theta}_{i}^{*}\right)+\varepsilon=\boldsymbol{\theta}_{i}^{* T} \boldsymbol{\xi}\left(\bar{X}_{i}\right)+\varepsilon_{i},(i=1,2)
$$

The optimal approximation parameters $\boldsymbol{\theta}_{i}^{*}$ are defined in the fuzzy system as follows:

$$
\begin{aligned}
& \boldsymbol{\theta}_{i}^{*}=\arg \min _{\boldsymbol{\theta} \in \Omega_{\theta_{i}}}\left(\sup _{\bar{X}_{i} \in U}\left|\hat{f}_{i}-\hat{\tau}_{i}\left(\bar{X}_{i}, \boldsymbol{\theta}_{i}^{*}\right)\right|\right) \\
& \Omega_{\theta_{i}}=\left\{\boldsymbol{\theta}_{i} \mid \boldsymbol{\theta}_{i}^{T} \boldsymbol{\theta}_{i} \leq M_{\theta_{i}}, M_{\theta_{i}}>0\right\}
\end{aligned}
$$

where $\Omega_{\theta_{i}}$ and $U$ are bounded compact sets of adjustable parameters $\boldsymbol{\theta}_{i}$ and fuzzy input vector $\bar{X}_{i} \cdot \mathcal{E}_{i}$ is a optimal approximation error of the fuzzy logic system. Generally, it is assumed that the optimal approximation error is bounded, satisfying $\left|\varepsilon_{i}\right| \leq \bar{\varepsilon}_{i}$.
Based on the above fuzzy logic systems, the adaptive fuzzy backstepping law is formulated as

$$
u=-\lambda_{2}\left(X_{2}-\hat{\boldsymbol{\theta}}_{1}^{T} \boldsymbol{\xi}\left(\bar{X}_{1}\right)\right)-z_{1}-\hat{\boldsymbol{\theta}}_{2}^{T} \boldsymbol{\xi}\left(\bar{X}_{2}\right)
$$

The corresponding differences between the optimal and estimated parameters are defined $\tilde{\boldsymbol{\theta}}=\boldsymbol{\theta}^{*}-\hat{\boldsymbol{\theta}}$, then the parameter adaptation laws for the fuzzy systems are chosen as

$$
\dot{\hat{\boldsymbol{\theta}}}_{i}=r_{i} z_{i} \xi\left(\bar{X}_{i}\right)-2 k_{i} \hat{\boldsymbol{\theta}}_{i},(i=1,2)
$$

where $r_{i}$ and $k_{i}$ are positive constants.

The stability analysis is summarized in theorem 1.

Theorem 1. Consider the actual dynamic models of the underactuated ships in presence of parameter variations and external disturbances represented by Eq. (1). The ideal dynamic equilibrium states reference trajectories are designed as Eq. (7). IF the adaptive fuzzy backstepping control law is designed as Eq. (22) with the adaptation laws showed in Eq. (23), then the stability of the entire adaptive fuzzy control system can be guaranteed. The parameter $\hat{\boldsymbol{\theta}}_{i}$ of the fuzzy logic system will remain bounded, and the tracking errors asymptotically converge to a neighborhood of zero.

Proof. Define the second Lyapunov function candidate as

$$
V=V_{2}+\sum_{i=1}^{2} \frac{1}{2 r_{i}} \tilde{\boldsymbol{\theta}}_{i}^{T} \tilde{\boldsymbol{\theta}}_{i}
$$

The derivative of Eq. (24) with respect to time can be represented as

$$
\begin{aligned}
\dot{V}= & \dot{V}_{2}-\sum_{i=1}^{2} \frac{1}{r_{i}} \tilde{\boldsymbol{\theta}}_{i}^{T} \dot{\hat{\boldsymbol{\theta}}}_{i} \\
= & -\sum_{i=1}^{2} \lambda_{i} z_{i}^{2}+\sum_{i=1}^{2} z_{i}\left(\hat{f}_{i}-\tau_{i}\right)+\sum_{i=1}^{2} \frac{1}{b_{i}} z_{i} \omega_{i}-\sum_{i=1}^{2} \frac{1}{r_{i}} \tilde{\boldsymbol{\theta}}_{i}^{T} \dot{\hat{\boldsymbol{\theta}}}_{i} \\
= & -\sum_{i=1}^{2} \lambda_{i} z_{i}^{2}+\sum_{i=1}^{2} z_{i}\left(\hat{f}_{i}-\boldsymbol{\theta}_{i}^{* T} \boldsymbol{\xi}\left(\bar{X}_{i}\right)\right) \\
& +\sum_{i=1}^{2} z_{i} \tilde{\boldsymbol{\theta}}_{i}^{T} \boldsymbol{\xi}\left(\bar{X}_{i}\right)-\sum_{i=1}^{2} \frac{1}{r_{i}} \tilde{\boldsymbol{\theta}}_{i}^{T} \dot{\hat{\boldsymbol{\theta}}}_{i}+\sum_{i=1}^{2} \frac{1}{b_{i}} z_{i} \omega_{i} \\
\leq & -\sum_{i=1}^{2} \lambda_{i} z_{i}^{2}+\sum_{i=1}^{2} \tilde{\boldsymbol{\theta}}_{i}^{T}\left(z_{i} \boldsymbol{\xi}\left(\bar{X}_{i}\right)-\frac{1}{r_{i}} \dot{\hat{\boldsymbol{\theta}}}_{i}\right) \\
& +\sum_{i=1}^{2}\left|z_{i} \overline{\boldsymbol{\theta}}_{i}\right|+\sum_{i=1}^{2} \frac{1}{b_{i}} z_{i} \omega_{i}
\end{aligned}
$$


We define

$$
a_{i}=\lambda_{i}-\frac{1}{2}-\frac{1}{2 \rho^{2} b_{i}^{2}},
$$

then

$$
\lambda_{i}=a_{i}+\frac{1}{2}+\frac{1}{2 \rho^{2} b_{i}^{2}}
$$

Substituting Eq. (27) into Eq. (25), then one can obtain

$$
\begin{aligned}
\dot{V} & \leq-\sum_{i=1}^{2} a_{i} z_{i}^{2}+\sum_{i=1}^{2} \tilde{\boldsymbol{\theta}}_{i}^{T}\left(z_{i} \xi\left(\bar{X}_{i}\right)-\frac{1}{r_{i}} \dot{\hat{\boldsymbol{\theta}}}_{i}\right) \\
& -\frac{1}{2} \sum_{i=1}^{2} z_{i}^{2}-\sum_{i=1}^{2} \frac{1}{2 \rho^{2} b_{i}^{2}} z_{i}^{2}+\sum_{i=1}^{2}\left|z_{i} \bar{\varepsilon}_{i}\right|+\sum_{i=1}^{2} \frac{1}{b_{i}} z_{i} \omega_{i}
\end{aligned}
$$

Because

$$
\begin{gathered}
-\frac{1}{2} \sum_{i=1}^{2} z_{i}^{2}+\sum_{i=1}^{2}\left|z_{i} \bar{\varepsilon}_{i}\right| \leq \frac{1}{2} \sum_{i=1}^{2} \bar{\varepsilon}_{i}^{2}, \\
-\sum_{i=1}^{2} \frac{1}{2 \rho^{2} b_{i}^{2}} z_{i}^{2}+\sum_{i=1}^{2} \frac{1}{b_{i}} z_{i} \omega_{i} \leq \sum_{i=1}^{2} \frac{1}{2} \rho^{2} \omega_{i}^{2},
\end{gathered}
$$

the adaptation laws for two fuzzy estimators are chosen as Eq. (23), Eq. (28) can be rewritten as follows:

$$
\begin{aligned}
\dot{V} \leq & -\sum_{i=1}^{2} a_{i} z_{i}^{2}+\sum_{i=1}^{2} \frac{k_{i}}{r_{i}}\left(2 \boldsymbol{\theta}_{i}^{* T} \hat{\boldsymbol{\theta}}_{i}-2 \boldsymbol{\theta}_{i}^{T} \hat{\boldsymbol{\theta}}_{i}\right) \\
& +\frac{1}{2} \sum_{i=1}^{2} \bar{\varepsilon}_{i}^{2}+\sum_{i=1}^{2} \frac{1}{2} \rho^{2} \omega_{i}^{2}
\end{aligned}
$$

Because $\boldsymbol{\theta}_{i}^{* T} \boldsymbol{\theta}_{i}^{*}+\hat{\boldsymbol{\theta}}_{i}^{T} \hat{\boldsymbol{\theta}}_{i} \geq 2 \boldsymbol{\theta}_{i}^{* T} \hat{\boldsymbol{\theta}}_{i}$, then

$$
2 \boldsymbol{\theta}_{i}^{* T} \hat{\boldsymbol{\theta}}_{i}-2 \hat{\boldsymbol{\theta}}_{i}^{T} \hat{\boldsymbol{\theta}}_{i} \leq \boldsymbol{\theta}_{i}^{* T} \boldsymbol{\theta}_{i}^{*}-\hat{\boldsymbol{\theta}}_{i}^{T} \hat{\boldsymbol{\theta}}_{i}
$$

Substituting Eq. (30) into Eq. (29), we get

$$
\begin{aligned}
\dot{V} & \leq-\sum_{i=1}^{2} a_{i} z_{i}^{2}+\sum_{i=1}^{2} \frac{k_{i}}{r_{i}}\left(-\hat{\boldsymbol{\theta}}_{i}^{T} \hat{\boldsymbol{\theta}}_{i}-\boldsymbol{\theta}_{i}^{* T} \boldsymbol{\theta}_{i}^{*}\right) \\
& +\sum_{i=1}^{2} \frac{2 k_{i}}{r_{i}} \boldsymbol{\theta}_{i}^{* T} \boldsymbol{\theta}_{i}^{*}+\frac{1}{2} \sum_{i=1}^{2} \bar{\varepsilon}_{i}^{2}+\sum_{i=1}^{2} \frac{1}{2} \rho^{2} \omega_{i}^{2}
\end{aligned}
$$

Because

$$
\tilde{\boldsymbol{\theta}}_{i}^{T} \tilde{\boldsymbol{\theta}}_{i}=\left(\boldsymbol{\theta}_{i}^{*}-\hat{\boldsymbol{\theta}}_{i}\right)^{T}\left(\boldsymbol{\theta}_{i}^{*}-\hat{\boldsymbol{\theta}}_{i}\right) \leq 2 \boldsymbol{\theta}_{i}^{* T} \boldsymbol{\theta}_{i}^{*}+2 \hat{\boldsymbol{\theta}}_{i}^{T} \hat{\boldsymbol{\theta}}_{i},
$$

then

$$
-\frac{1}{2} \tilde{\boldsymbol{\theta}}_{i}^{T} \tilde{\boldsymbol{\theta}}_{i} \geq-\hat{\boldsymbol{\theta}}_{i}^{T} \hat{\boldsymbol{\theta}}_{i}-\boldsymbol{\theta}_{i}^{* T} \boldsymbol{\theta}_{i}^{*}
$$

Substituting Eq. (33) into Eq. (31) and considering the assumption 1, Eq. (31) can be rewritten as follows:

$$
\begin{aligned}
\dot{V} & \leq-\sum_{i=1}^{2} a_{i} \frac{2 b_{i m}}{2 b_{i}} z_{i}^{2}-\sum_{i=1}^{2} \frac{k_{i}}{2 r_{i}} \tilde{\boldsymbol{\theta}}_{i}^{T} \tilde{\boldsymbol{\theta}}_{i} \\
& +\sum_{i=1}^{2} \frac{k_{i}}{2 r_{i}} \boldsymbol{\theta}_{i}^{* T} \boldsymbol{\theta}_{i}^{*}+\frac{1}{2} \sum_{i=1}^{2} \bar{\varepsilon}_{i}^{2}+\sum_{i=1}^{2} \frac{1}{2} \rho^{2} \omega_{i}^{2}
\end{aligned}
$$

Let $\lambda_{i} \geq \frac{1}{2}+\frac{1}{2 \rho^{2} b_{i}^{2}}, a_{i}>0$, we define:

$$
\begin{aligned}
& a_{0}=\min \left\{2 b_{i m} a_{i}, k_{i}, i=1,2\right\}, \\
& b_{0}=\sum_{i=1}^{2} \frac{2 k_{i}}{r_{i}} \boldsymbol{\theta}_{i}^{* T} \boldsymbol{\theta}_{i}^{*}+\frac{1}{2} \sum_{i=1}^{2} \bar{\varepsilon}_{i}^{2},
\end{aligned}
$$

Considering assumptions 2, we define

$$
c_{0}=\sum_{i=1}^{2} \frac{1}{2} \rho^{2} c_{i}
$$

then

$$
\begin{aligned}
\dot{V} & \leq-a_{0}\left(\sum_{i=1}^{2} \frac{1}{2 b_{i}} z_{i}^{2}+\sum_{i=1}^{2} \frac{1}{2 r_{i}} \tilde{\boldsymbol{\theta}}_{i}^{T} \tilde{\boldsymbol{\theta}}_{i}\right)+b_{0}+c_{0} \\
& =a_{0} V+b_{0}+c_{0}
\end{aligned}
$$

The general solution of Eq. (38) can be represented as

$$
\begin{aligned}
V(t) & \leq\left(V(0)-\frac{b_{0}+c_{0}}{a_{0}}\right) \exp \left(-a_{0} t\right)+\frac{b_{0}+c_{0}}{a_{0}} \\
& \leq V(0) \exp \left(-a_{0} t\right)+\frac{b_{0}+c_{0}}{a_{0}} \\
& \leq V(0)+\frac{b_{0}+c_{0}}{a_{0}}, \forall t \geq 0
\end{aligned}
$$

where $V(0)$ is the initial value of $V$.

Define a compact set $\Omega_{0}=\left\{X \mid V(X) \leq C_{0}\right\}$, where $C_{0}=V(0)+\frac{b_{0}+c_{0}}{a_{0}}$. From Eq. (24), we can come to the conclusion that the all signals of the close loop system are bounded, i.e. $\left(z_{1}, z_{2}, \tilde{\boldsymbol{\theta}}_{1}, \tilde{\boldsymbol{\theta}}_{2}\right)^{T} \in \Omega_{0}$.

\section{Simulation results}

In this section, some numerical simulations are performed to illustrate the effectiveness of our proposed control laws given by Eq. (22) and Eq. (23). 
Let the input fuzzy variables $X_{1}$ and $X_{2}$ be fuzzily partitioned into nine fuzzy sets, Negative Very Big (NVB), Negative Big (NB), Negative Medium (NM), Negative Small (NS), Zero (Z), Positive Small (PS), Positive Medium (PM), Positive Big (PB) and Positive Very Big (PVB). For simplicity, the means of the Gaussian functions are set at $-2,-1.5,-1,-0.5,0,0.5,1$, 1.5 and 2, respectively, and all the standard derivations of the Gaussian membership functions are set at 1 . However, no matter how many rules are activated, the fuzzification and defuzzification processes will derive the right control power when the fuzzy sets are sensible. Their values will be tuned online. So, the overlap region of fuzzy sets will not impact the performance of the controller. ${ }^{17}$

The Gaussian membership function of fuzzy sets can be defined as ${ }^{16}$

$$
\begin{gathered}
\mu_{F_{i}^{\prime}}\left(X_{i}\right)=\exp \left[-0.5\left(X_{i}+(l-5) * 0.5\right)^{2}\right] \\
(i=1,2 \quad l=1,2, \cdots, 9)
\end{gathered}
$$

Then:

$$
\begin{gathered}
\xi\left(\bar{X}_{1}\right)=\frac{\mu_{F_{1}^{j}}\left(X_{1}\right)}{\sum_{j=1}^{9} \mu_{F_{1}^{j}}\left(X_{1}\right)} \\
\xi\left(\bar{X}_{2}\right)=\frac{\mu_{F_{1}^{j}}\left(X_{1}\right) \mu_{F_{2}^{j}}\left(X_{2}\right)}{\sum_{j=1}^{9} \mu_{F_{1}^{j}}\left(X_{1}\right) \mu_{F_{2}^{j}}\left(X_{2}\right)}
\end{gathered}
$$

The Gaussian membership function of $X_{i}$ can be shown as Fig. 3.

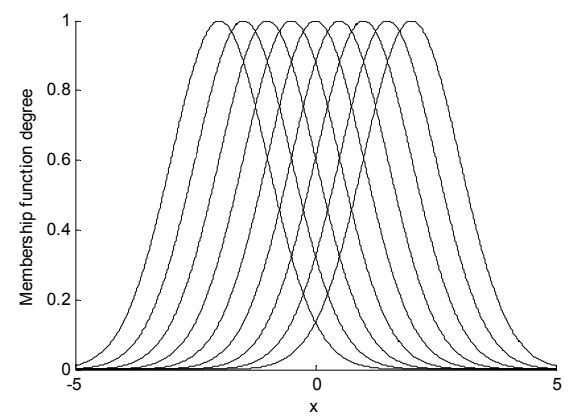

Fig. 3 The Gaussian membership function of $X_{i}$

The ship parameters are taken from Ref. 5 as follows: $U=8, K=0.4963, T=208.91$, and $\alpha=30$. The cruise speed variation is $\Delta U=0.2 \sin (2 \pi t)$. The initial condition for $y_{0}, \varphi_{0}$ and $r_{0}$ are $\boldsymbol{x}_{0}=\left[\begin{array}{lll}50 & 0.2 & 0\end{array}\right]$.The design parameters of the controller are: $\rho=1, \lambda_{1}=20, \lambda_{2}=300, k=0.002$, $k_{1}=k_{2}=2$ and $r_{1}=r_{2}=1.5$ 。

The four different cases are chosen as follows:

(i) If the stable time of the DES paths is set to be 200s, one can choose the weighting matrix of LQR for Eq. (7) as $R=1, \boldsymbol{Q}=\operatorname{diag}\left[\begin{array}{lll}1 & 1 & 0\end{array}\right]$. By calculation we obtain $\boldsymbol{K}=\left[\begin{array}{lll}1 & 4.42 & 1.1705\end{array}\right]$. The desired outputs are $x_{d}=500 \mathrm{~m}$, and $\varphi_{d}=0^{\circ}$. External disturbances are chosen random signals and signal intensity is two times. The simulation results are shown in Fig. 4. (a)

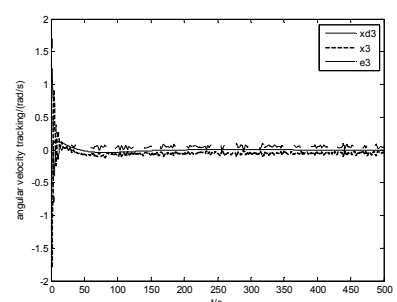

(c)

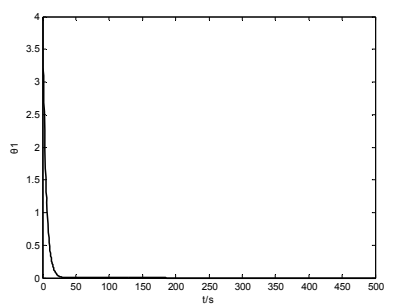

(e)

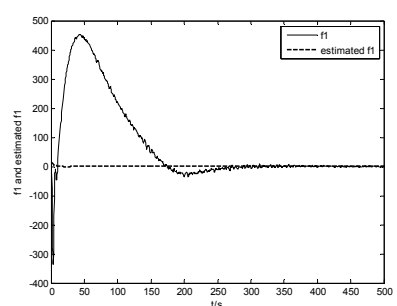

(g)

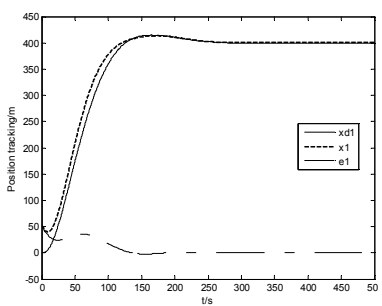

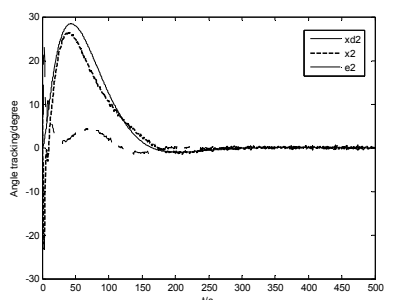

(b)

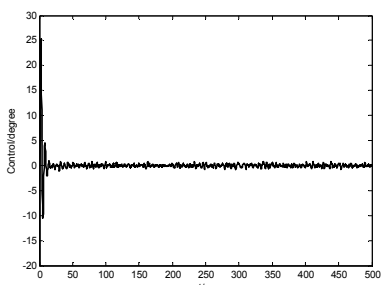

(d)

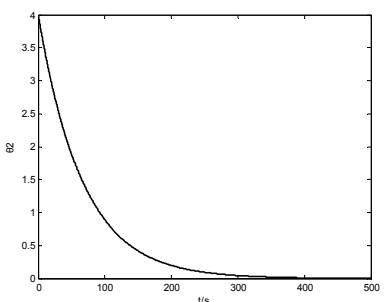

(f)

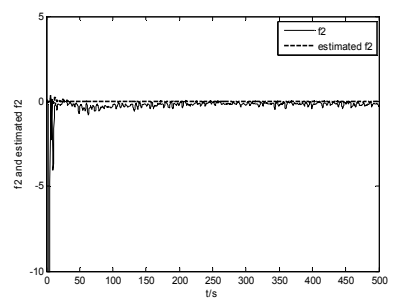

(h)
Fig. 4. Simulation results of the DES path tracking in case I. (a) sway displacement (b) yaw angle (c) yaw rate (d) control rudder angle (e) $\theta_{1}$ (f) $\theta_{2}$ (g) $f_{1}$ and estimated $f_{1}$ (h) $f_{2}$ and estimated $f_{2}$ 
Fig. 4 illustrates that the sway displacement $y$ and the yaw angle $\varphi$ can realize global asymptotic tracking under the action of control rudder angle in presence of parameters variation and random external disturbances. The tracking errors converge to zero at 200s.

(ii) If the stable time of the DES paths is shorten to 150s, one can choose $R=1, \boldsymbol{Q}=\operatorname{diag}\left[\begin{array}{lll}10 & 5 & 1\end{array}\right]$. By calculation we obtain $\boldsymbol{K}=\left[\begin{array}{lll}3.1623 & 8.4275 & 1.3047\end{array}\right]$. The desired output are:

$$
\left\{\begin{array}{l}
x_{d}=300 m, 0 \leq t \leq 250 s \\
x_{d}=500 m, 250 \leq t \leq 500 s .
\end{array}\right.
$$

The external disturbance signals are chosen as $\omega_{1}(t)=0.4 \sin (\pi t) \quad$ and $\quad \omega_{2}(t)=0.8 \sin (\pi t)$. The simulation results are shown in Fig. 5.

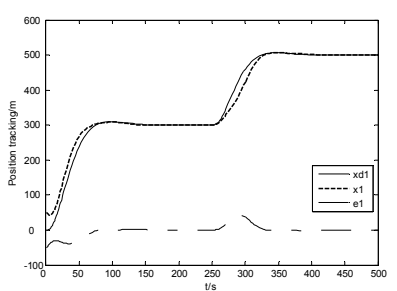

(a)

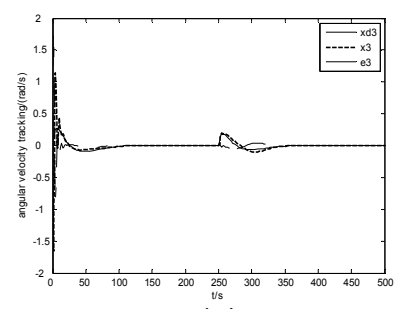

(c)

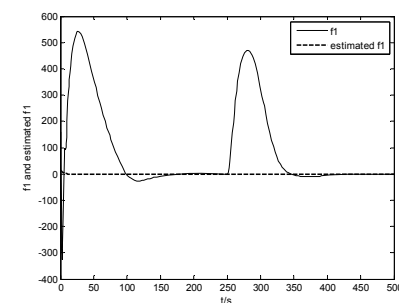

(e)

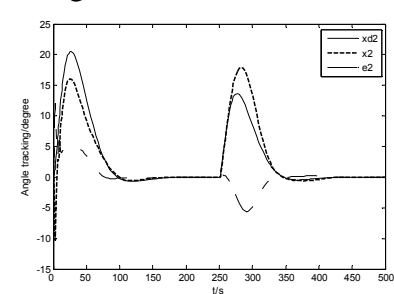

(b)

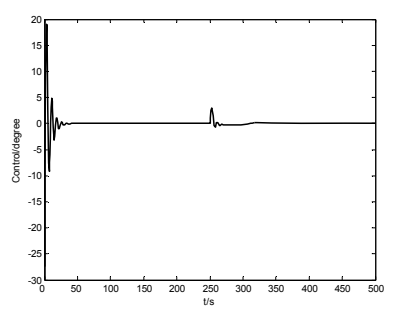

(d)

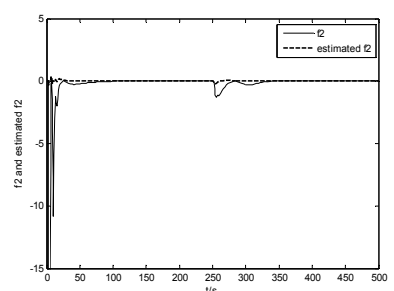

(f)
Fig. 5. Simulation results of the DES path tracking in case II. (a) sway displacement (b) yaw angle (c) yaw rate (d) control rudder angle (e) $f_{1}$ and estimated $f_{1}$ (f) $f_{2}$ and estimated $f_{2}$

From Fig. 5, it is concluded that the sway displacement and the yaw angle can rapidly adjust and track the new desired paths when the desired paths are changed suddenly at $t=250 \mathrm{~s}$. (iii) External disturbances are chosen pulse interferences added at 200s, 300s and 400s respectively. The pulse amplitude is $2 \% \mathrm{~s}$. The simulation results are shown in Fig. 6.

Fig. 6 shows that the sway displacement and yaw angle can rapidly adjust when there exist the pulse interferences, and the tracking control precision is also high. To compensate for the pulse perturbation, the control rudder angle changes around 5 degrees. Contrasted with Ref. 2 and 5, current disturbance $\omega_{1}$ was not considered in Ref. 2 and 5, so the controller can't resist the error of the sway displacement induced by the current disturbance.

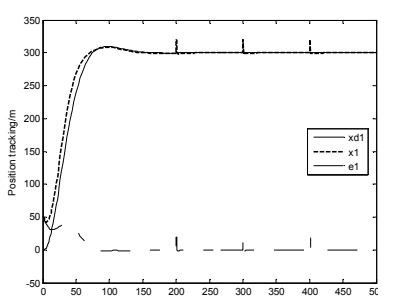

(a)

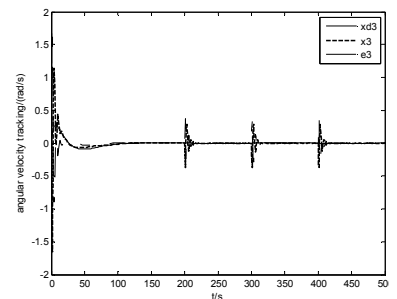

(c)

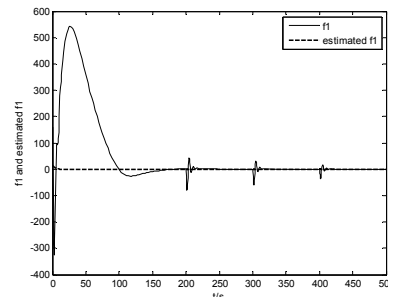

(e)

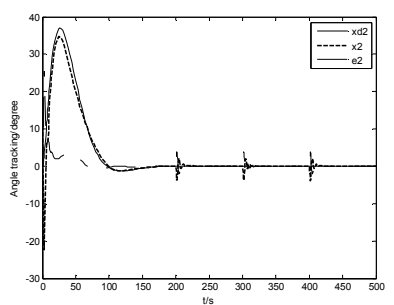

(b)

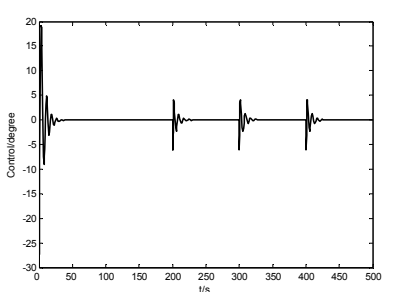

(d)

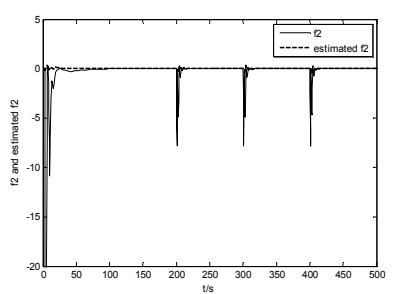

(f)
Fig. 6. Simulation results of the DES trajectories tracking in case III. (a) sway displacement (b) yaw angle (c) yaw rate (d) control rudder angle (e) $f_{1}$ and estimated $f_{1}$ (f) $f_{2}$ and estimated $f_{2}$

(iv) In order to verify the strong robustness of the proposed adaptive fuzzy control method (AFC), the adaptive dissipative control method (ADC) in Ref. 5 is chosen to compare with it. The ideal DES paths and uncertainties are chosen in case (i). The simulation 
results of two approaches are demonstrated in Fig. 7. Solid lines represent the curves of the ideal DES paths; dashed lines represent that of the AFC method; dashdoted lines represent that of the ADC method.

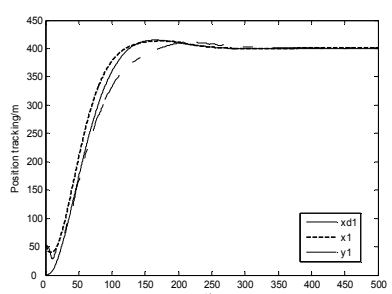

(a)

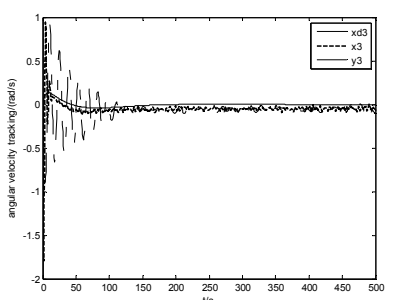

(c)

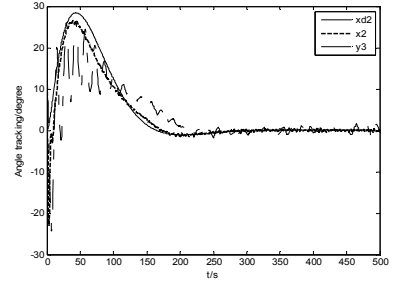

(b)

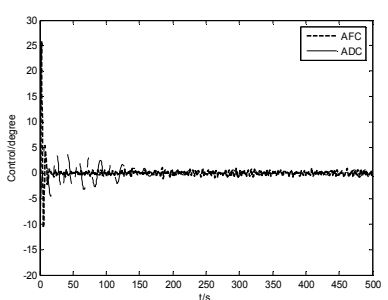

(d)
Fig. 7. Comparison of the two methods in case I. (a) sway displacement (b) yaw angle (c) yaw rate (d) control rudder angle

It is clear that the stable time is about 200s in the proposed control method while it is about 300s in the adaptive dissipative method for same ideal the DES paths. The fluctuation of all state variables and overshoot become bigger than that of the proposed control methods. One can find that the proposed method has the stronger robustness and the adaptability to the uncertainties.

As is shown above, the proposed scheme is effective and robust, and the control rudder angle is reasonable.

\section{Conclusions}

An adaptive fuzzy backstepping control approach based on the DES theory is proposed for path tracking of the underactuated ships. The ideal DES reference paths are designed by LQR control approach. By combining the DES tracking control method with the backstepping technology, the virtual control is treated as the DES and the original concept of the virtual control is got rid of so that it is easier to understand the control design. The numerical simulation results indicate the effectiveness and robustness of the proposed controller. Compared with the adaptive dissipative method, it is clear that the proposed adaptive fuzzy control approach is more efficient in performing path tracking in presence of parameters variation and external disturbance.

\section{Acknowledgements}

This work was supported by the National Science Foundation of China ( 60905051).

\section{References}

1. G. Chen, W. Yang, F. C. Sun, et al. Survey for motion control of underactuated surface vessels. Control and Decision, 24(3) (2009) 321-329.

2. G. Zhou, Q. H. Yao, Y. B. Chen, et al. A Study of Stability of Straight-line Tracking Control System for Underactuated Ship. Acta Automatica Sinica, 33(4) (2007) 378-384.

3. R. J, Zhang, Y. B. Chen, Z. Q. Sun, et al. Path control of a surface ship in restricted waters using sliding mode, IEEE Transactions on Control Systems Technology, 8(4) (2000) $722-732$.

4. K. D. Do, J. Pan. Underactuated Ships Follow Smooth Paths With Integral Actions and Without Velocity Measurements for Feedback: Theory and Experiments, IEEE Transactions on Control Systems Technology, 14(2) (2006) 308-322.

5. T. S. Li, Y. S. Yang, B. G. Hong. Adaptive robust dissipative designs on straight path control for underactuated ships, J. of Systems Engineering and Electronics, 17(1) (2006) 177-181.

6. L. Consolini, M. Tosques. How to find a minimum phase output in the exact tracking problem for the nonminimum phase underactuated surface ship, in Proc. of 49th IEEE Conference on Decision and Control (Altanta, USA, 2010), pp. 6236-6241.

7. W. L. Luo, Z. J. Zou, T. S. Li. Robust tracking control of nonlinear ship steering, Control Theory \& Applications, 26(8) (2009), 893-895.

8. Q. L Wang, Y. Chen. Equilibrium Control Theory and Direct Method of Feedback Linearization for Nonlinear Time Varying Systems, J. of Beijing Institute of Technology, 8(3) (1999) 306-311.

9. L. Wang, Q. L Wang. The nonlinear system tracking design based on the dynamic equilibrium state theory, in Proc. of the 25th Chinese Control Conf., (Herbin, China, 2006), pp. 143-146.

10. H. Jin, L. Wang. The nonlinear system design technique based on the dynamic equilibrium state theory, in Proc. of the 2th Innovative Computing, Information and Control, (Kumamoto, Japan, 2007), pp. 514-517.

11. L X Wang. Stable adaptive fuzzy control of nonlinear systems, IEEE Transactions on Fuzzy Systems, 2(1) (1993) 146-155. 
D. H. Qiu et al.

12. J. L. Castro. Fuzzy logic controllers are universal approximators, IEEE Transactions on Systems, Man and Cybernetics, 25(4) (1995) 629-635.

13. W. H. Wang, Y. Mao, J. Jing, et al. Driver's various information process and multi-ruled decision-making mechanism: a fundamental of intelligent driving shaping model. Int. J. of Computational Intelligence Systems, 4(3) (2011) 297-305

14. L. Yonggon, H. S. Zak. Uniformly ultimately bounded fuzzy adaptive tracking controllers for uncertain systems, IEEE Transactions on Fuzzy Systems, 12(6) (2004) 797811.

15. J. K. Liu. Design of Robot Control System and MATLAB Simulation. (Tsinghua University Press, Beijing, 2008).

16. V. L. Hoang. A note on the rate of uniform approximation of fuzzy systems, Int. J. of Computational Intelligence Systems, 4(4) (2011) 712-727.

17. C. Y. Chang. Adaptive fuzzy controller of the overhead cranes with nonlinear disturbance, $J$. of IEEE Transactions on Industrial Electronics, 3(2) (2007) 164172 